\title{
Semantics-aware Recommender Systems Exploiting Linked Open Data and Graph-based Features
}

\author{
Cataldo Musto \\ Dept. of Computer Science - University of Bari Aldo Moro \\ cataldo.musto@uniba.it \\ Marco de Gemmis \\ Dept. of Computer Science - University of Bari Aldo Moro \\ marco.degemmis@uniba.it
}

\begin{abstract}
In this contribution we propose a hybrid recommendation framework based on classification algorithms such as Random Forests and Naive Bayes, which are fed with several heterogeneous groups of features. We split our features into two classes: classic features, as popularity-based, collaborative and content-based ones, and extended features gathered from the Linked Open Data (LOD) cloud, as basic ones (i.e. genre of a movie or the writer of a book) and graph-based features calculated on the ground of the different topological characteristics of the tripartite representation connecting users, items and properties in the LOD cloud. In the experimental session we evaluate the effectiveness of our framework on varying of different groups of features, and results show that both LODbased and graph-based features positively affect the overall performance of the algorithm, especially in highly sparse recommendation scenarios. Our approach also outperforms several state-of-the-art recommendation techniques, thus confirming the insights behind this research.

This extended abstract summarizes the content of the journal paper [7] published on Knowledge-based Systems.
\end{abstract}

\section{CCS CONCEPTS}

- Information systems $\rightarrow$ Recommender systems; Web data description languages; - Computing methodologies $\rightarrow$ Supervised learning by classification;

\section{KEYWORDS}

Recommender Systems, Machine Learning, Linked Open Data

\section{ACM Reference Format:}

Cataldo Musto, Pasquale Lops, Marco de Gemmis, and Giovanni Semeraro. 2018. Semantics-aware Recommender Systems Exploiting Linked Open Data and Graph-based Features. In WWW'18 Companion: The 2018 Web Conference Companion, April 23-27, 2018, Lyon, France. ACM, New York, NY, USA, 4 pages. https://doi.org/10.1145/3184558.3186233

This paper is published under the Creative Commons Attribution 4.0 International (CC BY 4.0) license. Authors reserve their rights to disseminate the work on their personal and corporate Web sites with the appropriate attribution.

WWW'18 Companion, April 23-27, 2018, Lyon, France

(c) 2018 IW3C2 (International World Wide Web Conference Committee), published under Creative Commons CC BY 4.0 License.

ACM ISBN 978-1-4503-5640-4/18/04.

https://doi.org/10.1145/3184558.3186233

\author{
Pasquale Lops \\ Dept. of Computer Science - University of Bari Aldo Moro \\ pasquale.lops@uniba.it \\ Giovanni Semeraro \\ Dept. of Computer Science - University of Bari Aldo Moro \\ giovanni.semeraro@uniba.it
}

\section{INTRODUCTION}

According to recent statistics ${ }^{1}, 150$ billions of RDF triples and almost 10,000 linked datasets are now available in the so-called LOD cloud: such RDF triples interconnect in a semantics-aware fashion the information covering many topical domains, such as geographical locations, people, books, films, music, and so on. The nucleus of such data is commonly represented by DBpedia [1], the RDF mapping of Wikipedia. This huge availability of semantics-aware machine-readable data attracted researchers and practitioners in the area of Content-based Recommender Systems (RS) [4], willing to investigate how such information can be exploited to improve the effectiveness of existing algorithms or to tackle several problems RSs typically suffer from.

In this article we investigate the impact of such exogenous knowledge on the performance of a hybrid recommendation framework based on classification techniques as Random Forests and Naive Bayes. In this work we followed the hybridization strategy which is typically referred to as feature combination [2], that is to say, we represented the items by means of different heterogeneous groups of features and we used this unique representation to feed the classifiers with training examples. Such a model is then exploited to classify new and unseen items as relevant or not relevant for the target user.

The features we used are roughly classified in two groups: classic features and extended ones. The features that are typically used in hybrid item representations, as unstructured content-based features, collaborative features and simple popularity-based ones, fall in the first group. Next, we extended the representation by introducing data points gathered from the LOD cloud, as basic structured features (as the genre of a movie or the writer of a book) and graph-based features, calculated by mining the different topological characteristics of the tripartite graph-based representation that connects users, items and properties in the LOD cloud.

In the experimental session we evaluated the effectiveness of our framework on varying of these sets of features, and results provided several interesting insights, since it emerged that the overall accuracy significantly benefits from the introduction of LOD-based and graph-based features. Moreover, the results we obtained also overcame several state-of-the-art recommendation techniques.

\footnotetext{
${ }^{1}$ http://stats.lod2.eu/
} 


\section{METHODOLOGY}

In this section we provide the details of our methodology, by introducing the groups of features we used to feed the classification algorithms and by describing our recommendation framework.

\subsection{Description of the Features and Recommendation Framework}

Popularity features. This set of features includes basic popularitybased information about the items, such as the number of ratings received by the item, the number of positive ratings received by the item and the ratio between positive ratings and the overall number of ratings.

This (tiny) group of features may seem trivial and not useful, but this kind of data is typically very informative for a recommendation task, since it gives information about how popular is a certain item among the users and how positive is their general opinion about it. As Cremonesi et al. already shown [3], non-personalized algorithms based on simple popularity measures can obtain performance comparable to that of more sophisticated techniques.

Collaborative features. This class of features models the information encoded in the user-item matrix which is typically exploited in collaborative filtering $(\mathrm{CF})$ algorithms. Differently from classical $\mathrm{CF}$ algorithms, that use the whole matrix to calculate the neighbors of the target user and to predict the items the user may be interested in, in our approach we are only interested in extracting the column vector modeling the ratings received by an item in order to include them in our hybrid item representation. Accordingly, the number of collaborative features we encoded for each item corresponds to the number of the rows of the matrix, that is to say, to the number of the users in the dataset.

The choice of including this set of features in our hybrid representation is quite straightforward, since $\mathrm{CF}$ algorithms and matrix factorization techniques tend to obtain very good performance especially when the sparsity of the original matrix is not high.

Content-based features. Textual content is another interesting source that can be exploited to provide items with useful and descriptive features. As an example, the plot of a movie contains several distinctive characteristics of the item, which can be extracted from such data.

However, textual descriptions are typically noisy, thus it is necessary to properly process such data by adopting Natural Language Processing (NLP) techniques before including them in our items representation. In our pipeline the content was first tokenized, then stop-words were removed and the entities occurring in the text were identified. Next, the remaining tokens were stemmed. In this case, the amount of features added to the model corresponds to the size of the vocabulary, that is to say, to the number of different tokens occurring in the description of all the items in the dataset.

LOD-based features. The first group of extended features includes structured basic properties gathered from the LOD cloud, as the genre of a movie or the author of a book. To gather LOD-based features we preliminarily carried out a mapping procedure to obtain the corresponding URI for each item in the dataset. The goal of the mapping procedure is to identify, for each available item, the corresponding element in the LOD cloud the item refers to. As an example, we associate the movie The Matrix with its corresponding
Table 1: Partial representation of the vector modeling the LOD-based features extracted from DBpedia for the movie The Matrix

\begin{tabular}{|c|c|}
\hline property - value & The Matrix \\
\hline dbo:director - dbr:The_Wachowski & 1 \\
\hline dbo:director - dbr:Mel_Gibson & 0 \\
\hline dbo: composer - dbr:Ennio_Morricone & 0 \\
\hline dct:subject - dbc:Dystopian_films & 1 \\
\hline dct:subject - dbc:American_Horror_movies & 0 \\
\hline ... & $\ldots$ \\
\hline dbo:producer - dbr:Joel_Silver & 1 \\
\hline
\end{tabular}

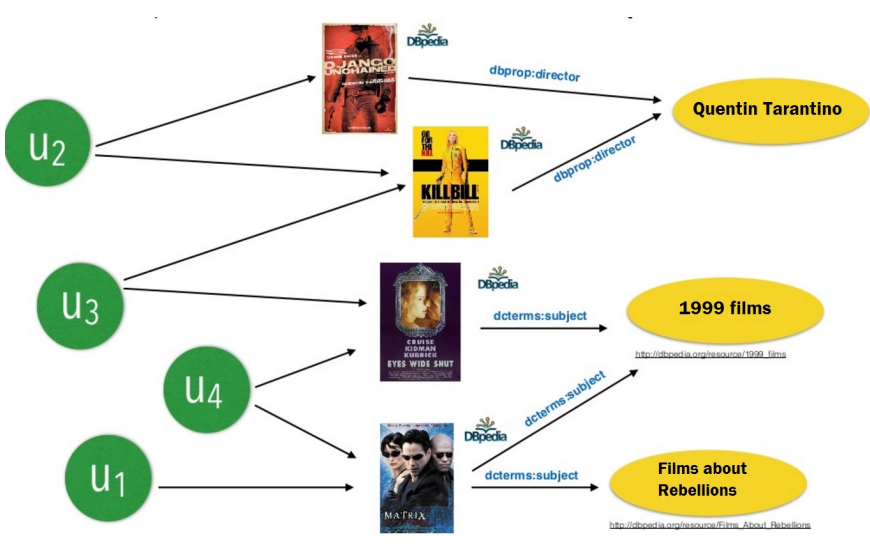

Figure 1: A toy example of a tripartite graph, modeling users, items and properties gathered from the LOD.

URI in the LOD cloud ${ }^{2}$. It is worth to emphasize that the mapping is a necessary and mandatory step to get an entry point to the LOD cloud.

Next, for each domain, we defined a subset of relevant properties by exploiting the outcomes of our previous research $[5,6]$ and finally we used SPARQL to extract such data.

As we did for content-based features, we built a vocabulary of LOD-based properties and we provided each item with these new features. The score of each feature was set to 1 if the item is described through that RDF property, 0 otherwise. Table 1 reports some of the properties describing The Matrix gathered from the LOD cloud. In this case, each feature is represented through the couple < property,value>, since each entity can have different roles in the same movie (and in different ones, as well).

Graph-based Features. The second group of extended features is built on the ground of the graph-based representation obtained by connecting the users to the items they liked and, in turn, the items to the properties gathered from DBPEDIA (see Figure 1). We refer to these features as tripartite ones.

Given such representations, we decided to mine this graph and to calculate some measures describing its topological characteristics. Specifically, in our item representation we encoded five graph-based features calculated on the tripartite user-item graph, that is to say:

${ }^{2}$ http://dbpedia.org/resource/The_Matrix 
Degree Centrality, Average Neighbor Degree, PageRank score, Node Redundancy and Cluster Coefficient.

Recommendation Framework. In this work we cast the recommendation task to a classification one, that is to say, we used the vectors representing the items the user liked as positive examples and those he did not like as negative examples. Next, we trained the classifiers and we exploited them to classify all the items the user did not consumed yet as interesting or not interesting for her.

To sum up, given a target user $u$, a training set $T R(u)$ (the items the user previously rated), and a group of features $F$, our classifier is fed with the examples $i_{F} \in T R(u)$ and we use the classification model to predict the most interesting items for the target user. Specifically, items in the test set are ranked according to the confidence of the prediction returned by the classification algorithm and the top- $K$ items are returned to the target user. In the experimental session the overall effectiveness of our recommendation framework has been evaluated by varying different sets of features and by using two different classification algorithms, namely Random Forests and Nä̈ve Bayes.

\section{EXPERIMENTAL EVALUATION}

Our experiments were designed on the ground of three different research questions: How do $L O D$-based features impact on the overall performance of the recommendations? (Experiment 1). How do graph-based features impact on the overall performance of the recommendations? (Experiment 2). How does our best-performing configuration perform with respect to state-of-the-art techniques? (Experiment 3).

Experimental protocol. Experiments were carried out on two state-of-the-art datasets, i.e. MovieLens $-1 \mathrm{M}^{3}$, and DBвоок. The first one is a widespread dataset for movie recommendation, the second was used in the ESWC 2014 Recommender Systems challenge ${ }^{4}$ and focuses on book recommendation.

Experiments were performed by adopting different protocols. We used a $80 \%-20 \%$ training-test split for MovieLens-1M. For DBbook we used the training-test split that provided with the data. Different protocols were also adopted to build user profiles. In MovieLens-1M, user preferences are expressed on a 5-point discrete scale, thus we decided to consider as positive only those ratings equal to 4 and 5. On the other side, the DBbook dataset is already available as binarized, thus no further processing was needed. As classification algorithms we used the implementations of Random Forest and Naive Bayes made available in the Weka Toolkit ${ }^{5}$.

Popularity features were extracted by simply processing the original data and by counting the ratings received by each item. As regards collaborative features, we replaced missing values with a special character and we used a binary representation to encode positive and negative ratings. Next, to generate content-based features we used the methods implemented in the Apache Lucene ${ }^{6}$ library for tokenization, language detection and stop-words removal. Textual descriptions were all gathered from the Wikipedia pages of

\footnotetext{
${ }^{3} \mathrm{http} / /$ grouplens.org/datasets/movielens/1m/

${ }^{4}$ http://challenges.2014.eswc-conferences.org/index.php/RecSys

${ }^{5} \mathrm{http}: / /$ www.cs.waikato.ac.nz/ml/weka/

${ }^{6} \mathrm{https} / / /$ lucene.apache.org/
}

Table 2: Impact of LOD-based Features on MovieLens data.

\begin{tabular}{|c|c|c|c|c|}
\cline { 2 - 5 } \multicolumn{1}{c|}{} & \multicolumn{2}{c|}{ RF } & \multicolumn{2}{c|}{ NB } \\
\hline \hline F1@5 & No-LOD & LOD & No-LOD & LOD \\
\hline \hline Popular (P) & 0.5338 & 0.5312 & 0.5458 & 0.5320 \\
\hline Collaborative (C) & 0.5618 & 0.5609 & 0.5486 & 0.5450 \\
\hline Content-based (T) & 0.4913 & $\mathbf{0 . 4 9 4 3}$ & 0.4913 & $\mathbf{0 . 4 9 3 2}$ \\
\hline \hline P+C & 0.5635 & $\mathbf{0 . 5 6 4 2 ( { } ^ { * } )}$ & 0.5483 & 0.5451 \\
\hline $\mathrm{P}+\mathrm{T}$ & 0.5051 & $\mathbf{0 . 5 0 7 9}$ & 0.4965 & $\mathbf{0 . 4 9 7 4}$ \\
\hline $\mathrm{C}+\mathrm{T}$ & 0.5187 & $\mathbf{0 . 5 1 8 8}$ & 0.5180 & 0.5169 \\
\hline \hline $\mathrm{P}+\mathrm{C}+\mathrm{T}$ & 0.5246 & 0.5246 & 0.5189 & 0.5174 \\
\hline
\end{tabular}

the items. Finally, tokens were stemmed by exploiting the Snowball library ${ }^{7}$.

As previously explained, each item was mapped to a DBpedia entry in order to gather the features from the LOD cloud. To this end, we exploited some mappings already available in literature. In our setting, 3,300 MovieLens-1M entries and 6,600 items (98.02\%) from DBbook ( $85 \%$ of the items) were successfully mapped. The items for which a DBpedia entry was not found were represented by using the basic groups of features alone. Finally, graph-based features were calculated by exploiting the Jung framework ${ }^{8}$, a Java library to manage graph-based data. As previously explained, for each item node we calculated Degree Centrality, Average Neighbor Degree, PageRank score, Node Redundancy and Cluster Coefficient for tripartite graph.

The performance of each configuration of our recommendation framework was evaluated in terms of F1@5, calculated through the Rival toolkit ${ }^{9}$.

Discussion of the Results. By analyzing the behavior of LOD features on MovieLens data (Table 2), it emerges that the only configuration that benefits of such injection is the one exploiting content-based features. This can be probably due to the low sparsity of the dataset, which makes superfluous most of the features except collaborative ones. However, even if these experimental settings showed that the adoption of LOD features has to be carefully evaluated, the overall best configuration (highlighted with $\left(^{*}\right)$ ) actually includes $L O D$ features, since the configuration merging popular, collaborative and LOD features obtained the higher F1@5. A similar pattern was noted on DBbook, since RF is the algorithm which takes the best from the LOD-based features. An interesting outcome emerging from this experiment is that when data are sparse, as for DBbook, LOD-based data points represent a good alternative also to collaborative features. Indeed, in this experiment Popular $+L O D$ obtained the best overall F1@5. This means that, when the rating patterns are noisy, LOD features can be used to enrich the representation with new and relevant information.

Next, we evaluated the impact of graph-based features on our recommendation framework. For each dataset we considered as baseline the best-performing configuration emerged from the previous tables and we extended the representation by introducing tripartite features. By considering MovieLens dataset, a positive

\footnotetext{
http://snowball.tartarus.org/

${ }^{8}$ http://jung.sourceforge.net/

${ }^{9}$ http://rival.recommenders.net/
} 
Table 3: Impact of LOD-based Features on DBbook data.

\begin{tabular}{|c|c|c|c|c|}
\cline { 2 - 5 } \multicolumn{1}{c|}{} & \multicolumn{2}{c|}{ RF } & \multicolumn{2}{c|}{ NB } \\
\hline \hline F1@5 & No-LOD & LOD & No-LOD & LOD \\
\hline \hline Popular (P) & 0.5610 & $\mathbf{0 . 5 6 5 9}\left(^{*}\right)$ & 0.5576 & $\mathbf{0 . 5 5 7 7}$ \\
\hline Collaborative (C) & 0.5421 & $\mathbf{0 . 5 5 6 0}$ & 0.5610 & 0.5564 \\
\hline Content-based (T) & 0.5532 & $\mathbf{0 . 5 5 5 1}$ & 0.5465 & $\mathbf{0 . 5 4 9 4}$ \\
\hline \hline P+C & 0.5627 & $\mathbf{0 . 5 6 3 0}$ & 0.5615 & 0.5580 \\
\hline $\mathrm{P}+\mathrm{T}$ & 0.5567 & $\mathbf{0 . 5 5 6 9}$ & 0.5467 & $\mathbf{0 . 5 4 9 7}$ \\
\hline $\mathrm{C}+\mathrm{T}$ & 0.5549 & $\mathbf{0 . 5 5 5 3}$ & 0.5464 & $\mathbf{0 . 5 4 9 1}$ \\
\hline \hline $\mathrm{P}+\mathrm{C}+\mathrm{T}$ & 0.5583 & 0.5560 & 0.5468 & $\mathbf{0 . 5 4 9 7}$ \\
\hline
\end{tabular}

Table 4: Impact of Graph-based Features.

\begin{tabular}{|c|c|c|c|c|}
\hline & \multicolumn{2}{|c|}{ MovieLens } & \multicolumn{2}{c|}{ DBbook } \\
\hline \hline & RF & NB & RF & NB \\
\hline \hline Baseline & 0.5635 & 0.5486 & 0.5627 & 0.5615 \\
\hline Baseline+Trip. & 0.5621 & 0.5483 & 0.5607 & 0.5542 \\
\hline \hline Baseline+LOD & 0.5642 & 0.5451 & 0.5659 & 0.5580 \\
\hline Baseline+LOD+Trip. & $\mathbf{0 . 5 6 7 8}\left(^{*}\right)$ & $\mathbf{0 . 5 4 8 1}$ & $\mathbf{0 . 5 6 6 7}\left({ }^{*}\right)$ & $\mathbf{0 . 5 5 8 9}$ \\
\hline
\end{tabular}

impact only emerged when graph-based features are merged with LOD-based ones. Indeed, both RF and NB are able to improve F1@5 with a statistically significant improvement when tripartite graphbased features are exploited. This means that the topological information coming from the injection of the features gathered from the LOD cloud can improve the performance of our framework. Overall, the best configuration for ML data is that based on both LOD-based and tripartite graph-based features which uses RF. Similar outcomes emerge if we take into account the results on DBbook data. Also in this case, when LOD-based features are included in the representation, graph-based features produce a significant increase of F1@5.

In the last experiment we compared the effectiveness of our hybrid recommendation methodology with several state of the art recommendation algorithms, as User-to-User (U2U-KNN), Item-toItem Collaborative Filtering (I2I-KNN), the Bayesian Personalized Ranking (BPRMF) and an implementation of PageRank with Priors. Moreover, we also compared our methodology to other LOD-aware recommendation techniques. As future work, we plan to compare our approach also to other semantics-aware RS [8]. Specifically, we used the features gathered from the LOD as side information for BPRMF and we also extended PageRank with Priors (PPR) with LOD-based features as we investigated in our previous research [5]. PPR was run by using default settings ( $80 \%$ of the weight distributed to the items the user liked). For brevity, we only report the results obtained by the best-performing configurations ( 80 neighbors for U2U-KNN and I2I-KNN, 100 factors for BPRMF, 50 factors for BPRMF with side information). For U2U-KNN, I2I-KNN and BPRMF we exploited the implementations already available in MyMediaLite ${ }^{10}$, while the methods implemented in the Jung framework ${ }^{11}$ were used to run PPR.

\footnotetext{
${ }^{10} \mathrm{http} / / / \mathrm{www} \cdot \mathrm{mym}$ edialite.net/

${ }^{11} \mathrm{http}: / /$ jung.sourceforge.net/
}

Table 5: Comparison to state of the art algorithms

\begin{tabular}{|c|c|c|}
\hline & \multicolumn{2}{|c|}{ F1@5 } \\
\hline Algorithm & MovieLens-1M & DBbook \\
\hline \hline LOD-RecSys & $\mathbf{0 . 5 6 7 8}$ & $\mathbf{0 . 5 6 6 7}$ \\
\hline \hline U2U-KNN & 0.4270 & 0.5193 \\
\hline I2I-KNN & 0.4320 & 0.5111 \\
\hline \hline BPRMF & 0.5218 & 0.5290 \\
\hline BPRMF+LOD & 0.5215 & 0.5304 \\
\hline \hline PPR & 0.5397 & 0.5502 \\
\hline PPR+LOD & 0.5400 & 0.5540 \\
\hline
\end{tabular}

As shown in Table 5, our hybrid recommendation framework always overcomes all the baselines on MovieLens-1M and DBbook data. All the increases are statistically significant. It is worth to note that our approach obtains better results when compared to both classic baselines as well as to other LOD-aware techniques as $B P R M F+L O D$ and PPR+LOD.

To sum up, several interesting outcomes emerge from these experiments: first, RF was the classification algorithm able to take the best out of our hybrid data representation. Another interesting outcome is the connection between the sparsity of the dataset and the choice of the features to be included in the model. When the dataset is not sparse, collaborative features along with nonpersonalized popularity-based emerge as the most informative ones. On the other side, when data are sparse, collaborative features need to be replaced or coupled with different information sources. These results further confirmed the outcomes behind this research, since they clearly showed that the injection of exogenous data points gathered from the LOD cloud (in the form of both semantics-aware content-based features and topological tripartite ones) can significantly improve the predictive accuracy of our recommendation framework, leading to an interesting improvement over all the state-of-the-art baselines.

\section{REFERENCES}

[1] S. Auer, C. Bizer, G. Kobilarov, J. Lehmann, R.Cyganiak, and Z. G. Ives. 2007. DBpedia: A Nucleus for a Web of Open Data. In ISWC 2007 (Lecture Notes in Computer Science), Vol. 4825. Springer, 722-735. DOI : http://dx.doi.org/10.1007/ 978-3-540-76298-0_52

[2] R. Burke. 2002. Hybrid recommender systems: Survey and experiments. UMUAI 12, 4 (2002), 331-370.

[3] P. Cremonesi, Y. Koren, and R. Turrin. 2010. Performance of recommender algorithms on top-n recommendation tasks. In ACM RECSYS. ACM, 39-46.

[4] M. de Gemmis, P. Lops, C. Musto, F. Narducci, and G. Semeraro. 2015. SemanticsAware Content-Based Recommender Systems. In Recommender Systems Handbook. Springer, 119-159.

[5] C. Musto, P. Basile, P. Lops, M. de Gemmis, and G. Semeraro. 2017. Introducing linked open data in graph-based recommender systems. Information Processing \& Management 53, 2 (2017), 405-435.

[6] C. Musto, P. Lops, P. Basile, M. de Gemmis, and G. Semeraro. Semantics-aware Graph-based Recommender Systems Exploiting Linked Open Data. In Proceedings of the 2016 Conference on User Modeling Adaptation and Personalization (UMAP 2016). ACM, 229-237.

[7] C. Musto, P. Lops, M. de Gemmis, and G. Semeraro. 2017. Semantics-aware Recommender Systems exploiting Linked Open Data and graph-based features. Knowledge-Based Systems 136, Supplement C (2017), 1 - 14.

[8] C. Musto, G. Semeraro, P. Lops, and M. de Gemmis. 2011. Random Indexing and Negative User Preferences for Enhancing Content-Based Recommender Systems. In EC-Web 2011 (Lecture Notes in Business Inf. Processing), Vol. 85. Springer, 270281 